\title{
Investigation on Tri-hexagonal Boron Nanotube by Exploiting the Certain Topological Indices and Their $M$-polynomials
}

\author{
V. Lokesha, Sushmitha Jain ${ }^{*}$, T. Deepika \\ Department of Studies in Mathematics, Vijayanagara Sri Krishnadevaraya University, Ballari, India \\ *Corresponding author: sushmithajain9@gmail.com
}

Received July 01, 2017; Revised August 15, 2017; Accepted September 21, 2017

\begin{abstract}
Due to the presence of multicenter bonds and their novel electronic properties, boron nanotubes are attractive. The tri-hexagonal boron nanotubes are build up from triangles and hexagons. It is useful to the QSPR/QSAR studies. Topological indices are classified in different forms such as, degree based topological indices, distance based topological indices and counting related topological indices etc. Here, we concentrated the reckoning of topological indices such as first zagreb, second zagreb, modified second zagreb index, generalized randic index, symmetric division degree index for the tri-hexagonal boron nanotube. Also, established their M-polynomials and using maple software plotted the 3D structure.
\end{abstract}

Keywords: Tri-hexagonal boron nanotube, topological indices, M-polynomials

Cite This Article: V. Lokesha, Sushmitha Jain, and T. Deepika, "Investigation on Tri-hexagonal Boron Nanotube by Exploiting the Certain Topological Indices and Their $M$-polynomials." Turkish Journal of Analysis and Number Theory, vol. 5, no. 6 (2017): 197-201. doi: 10.12691/tjant-5-6-1.

\section{Introduction}

Chemical graph theory is an important branch of graph theory as it contains discrete Adriatic indices. In this theory, a descriptor of a molecular graph $\mathrm{G}$ is a number related to the structure of $G$ and is invariant under the automorphisms of the graph.

Recently, D. Vukicevic [24] revealed the set of 148 discrete Adriatic indices. They were analyzed on the testing sets provided by the International Academy of Mathematical Chemistry (IAMC) and it had been shown that they have good predictive properties in many cases. There was a vast research regarding various properties of these topological indices.

There are two oldest degree based topological indices, first and second zagreb indices were introduced more than thirty years ago by Gutman and Trinajstic [11] which are defined as

$$
M_{1}(G)=\sum_{u v \in E(G)} d_{u}+d_{v}
$$

and

$$
M_{2}(G)=\sum_{u v \in E(G)} d_{u} d_{v}
$$

where $d_{u}$ denotes the degree of a vertex $u$ in $G$.

Both the first zagreb index and the second zagreb index give greater weights to the inner vertices and edges, and smaller weights to the outer vertices and edges, which opposes intuitive reasoning.

For a simple connected graph $G$, the second modified zagreb index [18] is defined as

$$
{ }^{m} M_{2}(G)=\sum_{u v \in E(G)} \frac{1}{d_{u} d_{v}} .
$$

Symmetric division deg index $[8,9]$ is one of the discrete Adriatic indices that is good predictor of total surface area for polychlorobiphenyls. The symmetric division degree index of a connected graph $G$ is defined as

$$
S D D(G)=\sum_{u v \in E(G)} \frac{d_{u}^{2}+d_{v}^{2}}{d_{u} d_{v}} .
$$

The Randi'c index [19] also the most popular, the most often applied and the most studied among all other topological indices. The Randi'c index denoted by $R(G)$ and introduced by Milan Randi'c in 1975, is also one of the oldest topological index.

$$
R(G)=\sum_{u v \in E(G)} \frac{1}{\sqrt{d_{u} d_{v}}} .
$$

In 1998, working independently, Bollobas and Erdos [2] and Amic et. al., [1] proposed the generalised Randi'c index and has been studied extensively by both chemists and mathematicians. The ordinary Randi'c connectivity index has been extended to the general Randi'c connectivity index defined as 


$$
R_{\alpha}(G)=\sum_{u v \in E(G)}\left(d_{u} d_{v}\right)^{\alpha} .
$$

The inverse sum index (ISI) is a significant predictor of total surface area for octane isomers these are introduced by Damir Vukicevic and Marija Gasperov [4].

$$
\operatorname{ISI}(G)=\sum_{u v \in E(G)} \frac{d_{u} d_{v}}{d_{u}+d_{v}} .
$$

Another variant of the Randi'c index is the harmonic index [21] defined as

$$
H(G)=\sum_{u v \in E(G)} \frac{2}{d_{u}+d_{v}} .
$$

The $M$-polynomial [14] of graph $G$ is defined as

$$
M(G, x, y)=\sum_{i \leq j} m_{i j}(G) x^{i} y^{j}
$$

where $m_{i j}(G),(i, j \geq 1)$ be the number of edges $e=$

\begin{tabular}{|c|c|c|}
\hline Topological index & $f(x, y)$ & Derivation from $M(G ; x, y)$ \\
\hline First zagreb & $x+y$ & $\left(D_{x}+D_{y}\right) M(G ; x, y) \mid x=y=1$ \\
\hline Second zagreb & $x y$ & $\left(D_{x} D_{Y}\right) M(G ; x, y) \mid x=y=1$ \\
\hline $\begin{array}{c}\text { Second modified } \\
\text { zagreb }\end{array}$ & $\frac{1}{x y}$ & $\left(S_{x} S_{Y}\right) M(G ; x, y) \mid x=y=1$ \\
\hline Randic & $(x y)^{\alpha}$ & $\left(D_{x}^{\alpha} D_{y}^{\alpha}\right) M(G ; x, y) \mid x=y=1$ \\
\hline $\begin{array}{c}\text { Symmetric division } \\
\text { degree }\end{array}$ & $\frac{x^{2}+y^{2}}{x y}$ & $\left(D_{x} S_{y}+D_{y} S_{x}\right) M(G ; x, y) \mid x=y=1$ \\
\hline Harmonic & $\frac{2}{x+y}$ & $2 J S_{x} M(G ; x, y) \mid x=1$ \\
\hline Inverse sum index & $\frac{x y}{x+y}$ & $J S_{x} D_{x} D_{y} M(G ; x, y) \mid x=1$ \\
\hline
\end{tabular}
$u v$ of $G$ such that $\left(d_{u}, d_{v}\right)=(i, j)$.

Table 1. Derivation of degree- based topological indices from M-polynomials

Where

$$
\begin{gathered}
D_{x}=\frac{\partial(f(x, y))}{\partial x}, D_{y}=\frac{\partial(f(x, y))}{\partial y}, S_{x}=\int_{0}^{x} \frac{f(t, y)}{t} d t, \\
\text { and } S_{x}=\int_{0}^{y} \frac{f(x, t)}{t} d t, J=f(x, x) .
\end{gathered}
$$

\section{Tri-hexagonal Boron Nanotube}

In 1991 nanotubes were discovery, it has created intense experimental and theoretical interest in such structures. Theoretical perspectives of carbon tubes suggest that their electrical properties will range from metallic to semiconducting, depending on the tube diameter.

In 2004 the first boron triangular nanotubes were innovated and evolved from a triangular sheet. The latest discovery of boron triangular nanotubes challenges the monopoly of carbon nanotubes (CNTs). Researchers believe that carbon nanotubes are less significant than boron triangular nanotubes. Eventually scientists have proved this speculation by innovating the world's smallest superconductor using nanoscale molecular superconducting boron wires [3]. As for as special features are concerned boron nanotubes also have some better features when compared to CNTs such as high chemical stability, high resistance to oxidation at high temperatures and are a stable wide band-gap semiconductor. Because of these properties, they can be used for applications at high temperatures or in corrosive environments such as batteries, fuel cells, super capacitors, high speed machines as solid lubricant.

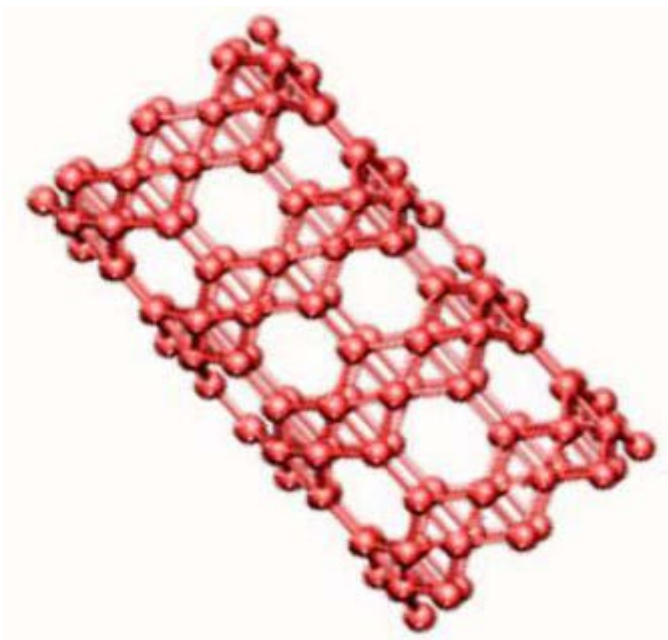

Figure 1. Three-dimensional perception of tri-hexagonal boron nanotube

The boron triangular nanotubes are evolved from CNTs by adopting an additional atom to the center of each hexagon. A new class of boron nanotubes which are constructed from triangles and hexagons, called the trihexagonal boron nanotube. These nanotubes are formed by removing some atoms from boron triangular nanotubes.

These nanotubes are sparser than the other boron nanotube and after relaxation it remains at and metallic independent of their chirality.

We denote this nanotube by $C_{3} C_{6}(H)[m ; n]$, where $m$ is the number of hexagons in one column and $n$ is the number of hexagons in one row in two-dimensional lattice of $C_{3} C_{6}(H)[m ; n]$ nanotube.

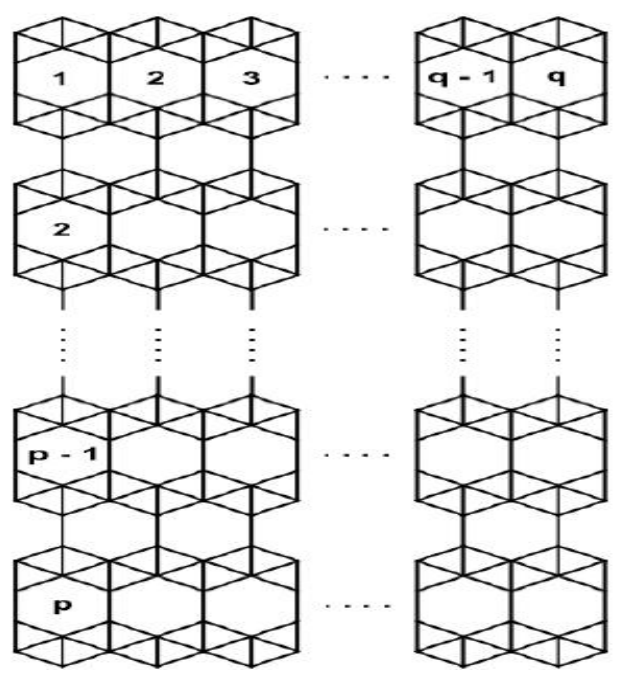

Figure 2. The graph of $C_{3} C_{6}(H)[m ; n]$ nanotube

Recently, I. Nadeem, H. Shaker [13] obtained the expressions for certain topological indices of tri-hexagonal boron nanotube. This motivates us to reckon on certain topological indices for the tri-hexagonal boron nanotube and structured their corresponding M-polynomials. Finally, 
Wrapping up 3D plot of concerned Topological indices utilized for Maple Software.

\section{Main Results}

In this section, we present our main results. In the following, we determine the topological indices and $M$-polynomials of the tri-hexagonal boron nanotube.

Theorem 1. Consider the tri-hexagonal boron nanotube $T=C_{3} C_{6}(H)[m ; n]$, then

1. $M_{1}(T)=2 n(82 m-7)$

2. $M_{2}(T)=2 n(186 m-23)$

3. ${ }^{m} M_{2}(G)=\frac{3 n}{400}(118 m+5$

4. $R(T)=2 m n\left(\frac{2+3 \sqrt{5}}{5}\right)+\sqrt{5} n\left(\frac{19 \sqrt{3}-36}{20 \sqrt{3}}\right)$

5. $S D D(T)=\frac{1}{10}(366 m n-5 n)$

6. $H(T)=\frac{1}{60}(318 m n-5 n)$

7. $\operatorname{ISI}(T)=\frac{1}{12}(488 m n+49 n)$.

Proof. Let the total number of vertices in tri hexagonal boron nanotube is $8 m n$ and $\mathrm{n}(18 \mathrm{~m}-1)$ number of edges.

Now, if we partition the edges correspond to their degree of end vertices which are

$$
\begin{aligned}
E_{(3,5)}=\left\{e=u v \in E(T) \mid d_{u}=3 \text { and } d_{v}=5\right\} \\
E_{(4,4)}=\left\{e=u v \in E(T) \mid d_{u}=4 \text { and } d_{v}=4\right\} \\
\rightarrow\left|E_{(4,4)}\right|=6(2 m-1)
\end{aligned}
$$

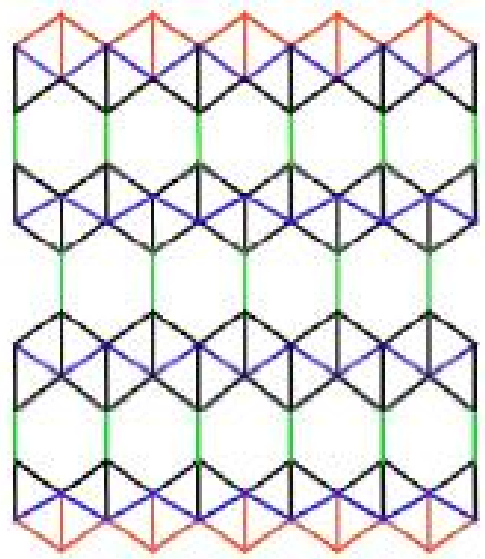

Figure 3. The graph of $C_{3} C_{6}(H)[m ; n]$ nanotube with $m=2$ and $n=5$
The representatives of these partite sets are shown in Figure 3 in which red, green, black and blue edges are edges belong to $E_{(3 ; 5)}, E_{(4 ; 4)}, E_{(4 ; 5)}$ and $E_{(5 ; 5)}$ respectively.

Now consider,

$$
\begin{aligned}
M_{1}(T)= & \left|E_{(3,5)}\right|\left(d_{u}+d_{v}\right)+\left|E_{(4,4)}\right|\left(d_{u}+d_{v}\right) \\
& +\left|E_{(4,5)}\right|\left(d_{u}+d_{v}\right)+\left|E_{(5,5)}\right|\left(d_{u}+d_{v}\right) \\
= & 2 n(82 m-7) . \\
M_{2}(T)= & \left|E_{(3,5)}\right|\left(d_{u} d_{v}\right)+\left|E_{(4,4)}\right|\left(d_{u} d_{v}\right) \\
& +\left|E_{(4,5)}\right|\left(d_{u} d_{v}\right)+\left|E_{(5,5)}\right|\left(d_{u} d_{v}\right) \\
= & 2 n(186 m-23) .
\end{aligned}
$$

Similarly, the proof is followed by the same technique by taking into account of edge partition and then applying Equations (3)-(8) to $T=C_{3} C_{6}(H)[m ; n]$, we come to the results as required.

Theorem 2. Let $\mathrm{T}$ be tri-hexagonal boron nanotube, then $M$-polynomial is

1. $M(T, x, y)=6 n x^{3} y^{5}+2 m n-n x^{4} y^{4}$

$+6 n(2 m-1) x^{4} y^{5}+4 m n x^{5} y^{5}$

2. $M_{1}(T)=(164 m n-14 n)$

3. $M_{2}(T)=(22 m n-n)(304 m n-40 n)$

4. ${ }^{m} M_{2}(T)=\frac{1}{800}\left(740 m^{2} n^{2}-3132 m n^{2}-215 n^{2}\right)$

5. $R(T)=(22 m n-n)^{\alpha}(304 m n-40 n)^{\alpha}$

6. $S D D(T)=\frac{1}{10}\left(2812 m^{2} n^{2}-560 m n^{2}+25 n^{2}\right)$

7. $H(T)=\frac{n^{2}}{20}\left(180 m^{2} n^{2}-764 m n^{2}-43\right)$

8. $\operatorname{ISI}(T)=\frac{1}{40}\left[\left(180 m^{2} n^{2}-764 m n^{2}-43\right)\right.$

$(22 m n-n)(304 m n-40 n)]$.

\section{Proof:}

Let

$$
\begin{aligned}
& M(T, x, y)=\sum_{i \leq y} m_{i j}(T) x^{i} y^{j} \\
&=\sum_{3 \leq 5} m_{35}(T) x^{3} y^{5}+\sum_{4=4} m_{44}(T) x^{4} y^{4} \\
&+\sum_{4 \leq 5} m_{45}(T) x^{4} y^{5}+\sum_{5=5} m_{55}(T) x^{5} y^{5} \\
&= \sum_{u v \in E(3,5)} m_{35}(T) x^{3} y^{5}+\sum_{u v \in E_{(4,4)}} m_{44}(T) x^{4} y^{4} \\
&+\sum_{u v \in E_{(4,5)}} m_{45}(T) x^{4} y^{5}+\sum_{u v \in E_{(5,5)}} m_{55}(T) x^{5} y^{5} \\
&=\left|E_{(3,5)}\right| x^{3} y^{5}+\left|E_{(4,4)}\right| x^{4} y^{4}+\left|E_{(4,5)}\right| x^{4} y^{5}+\left|E_{(5,5)}\right| x^{5} y^{5} \\
&= 6 n x^{3} y^{5}+2 m n-n x^{4} y^{4} 6 n(2 m-1) x^{4} y^{5}+4 m n x^{5} y^{5} .
\end{aligned}
$$

Now, from $M$-polynomial equation we compute the following

$$
\begin{aligned}
D_{x}= & 18 n x^{3} y^{5}+8 m n-4 n x^{4} y^{4} \\
& +24 n(2 m-1) x^{4} y^{5}+20 m n x^{5} y^{5}
\end{aligned}
$$




$$
\begin{array}{rl} 
& \left.D_{x}\right|_{x=y=1}=76 m n-10 n \\
D_{y}= & 30 n x^{3} y^{5}+8 m n-4 n x^{4} y^{4} \\
+ & 30 n(2 m-1) x^{4} y^{5}+20 m n x^{5} y^{5} \\
& \left.D_{y}\right|_{x=y=1}=88 m n-4 n \\
S_{x}= & \frac{6 n x^{3} y^{5}}{3}+\frac{2 m n-n x^{4} y^{4}}{4} \\
+ & \frac{6 n(2 m-1) x^{4} y^{5}}{4}+\frac{4 m n x^{5} y^{5}}{5} \\
& \left.S_{x}\right|_{x=y=1}=\frac{10 m n+43 n}{40} \\
S_{y}= & \frac{6 n x^{3} y^{5}}{5}+\frac{2 m n-n x^{4} y^{4}}{4} \\
+ & \frac{6 n(2 m-1) x^{4} y^{5}}{5}+\frac{4 m n x^{5} y^{5}}{5} \\
J=6 n x^{3} x^{5}+2 & m n-n x^{4} x^{4}+6 n(2 m-1) x^{4} x^{5}+4 m n x^{5} x^{5} \\
\left.J\right|_{x=1}=18 m n-n .
\end{array}
$$

Using Table 1 derivations of $M$-polynomial and the values of $D_{x}, D_{y}, S_{x}, S_{y}$ and $J$ we get the required results.

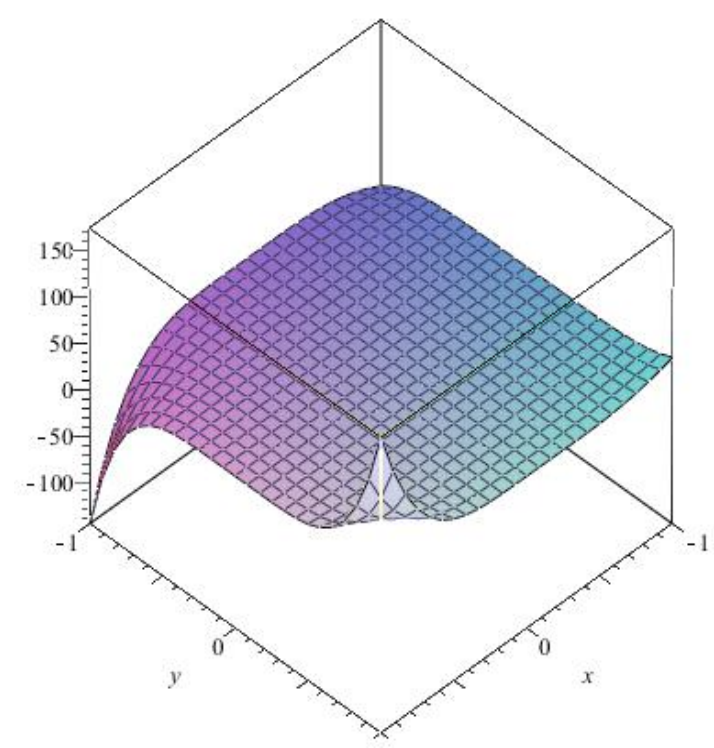

Figure 4. The graph of $M$ - polynomial of tri-hexagonal boron nanotube

\section{Conclusion}

In this paper, we computed the certain degree-based topological indices and $M$-polynomial. Also, plotted the $3 D$ structure for tri-hexagonal boron nanotube. The topological indices calculated in here can help us to understand the physical features, chemical reactivity, and biological activities. In this perspective, topological index is considered as vital role that maps each molecular structure to a real number and is used as a descriptor of the molecule under testing.

\section{Acknowledgements}

The second author is thankful to Maulana Azad National Fellowship, Ref.No.F117.1/201718/MANF201718KAR76148. First and third authors were thankful to Department of Science and Technology for research funding, Project No. SR/WOS-A/PM-43/2016.

\section{References}

[1] Amic. D., Beslo. D., Lucic. B., Nikolic. S. and Trinajstic. N, "The vertex connectivity index revisited”, J. Chem. Inf. Comput. Sci. 38, 819-822, 1998.

[2] Bollobas. B. and Erdos, "Graphs of extremal weights", Ars Combin., 50, 225-233, 1998.

[3] K. Clark, A. Hassanien, S. Khan, K. F. Braun, H. Tanaka and S. W. Hla, Nat. Nanotechnol., 5, 261, 2010.

[4] Damir Vukicevi and Marija Gasperov, "Bond additive modeling 1. Adriatic Indices”, Croat. Chem. Acta, 83(3), 243-260, 2010.

[5] Deutsch. E and Klavzar. S, "M-Polynomial, and degree-based topological indices", Iran. J. Math. Chem., 6, 93-102, 2015.

[6] J. Devillers and A. T. Balaban (Eds.), Topological Indices and Related Descriptors in QSAR and QSPR, Gordon and Breach, Amsterdam, 1999.

[7] G.H. Fath-Tabar "Old and new Zagreb index," Match-Commun. Math. Compute. Chem., 65, 79-84, 2011.

[8] C. K. Gupta, V. Lokesha and S. B. Shetty, "On the Symmetric division deg index of graph”, South East Asian Journal Of Mathematics, 4(11), 59-80, 2016.

[9] C. K. Gupta, V. Lokesha, S. B. Shetty and P. S. Ranjini, "Graph Operations on Symmetric Division Deg Index of Graph", Palestine Journal of Mathematics, 6(1), 280-286, 2017.

[10] Gutman. I. Some properties of the Wiener polynomials, Graph Theory Notes N. Y., 125, 13-18, 1993.

[11] Gutman. I. and Das. K. Ch., "The first zagreb indices 30 years after”, MATCH Commun. Math.Comput. Chem. 50, 83-92, 2004.

[12] F. Harary, Graph theory, Reading, MA: Addison-Wesley, 1994.

[13] Imran Nadeem, H. Shaker, "On topological indices of trihexagonal boron nanotubes", Journal of Optoelectronics and Advance Materials, 1810(9), 893-898, 2016.

[14] Deutsch. E, Klavzar. S, "M-Polynomial and degree-base topological indices”, Iran. J. Math. Chem., 6, 93-102, 2015.

[15] M. H. Khalifeha, H. Youse -Azari and A. R. Ashra, "The first and second Zagreb indices of some graph operations", Discrete Applied Mathematics, 157(4), 804-811, 2009.

[16] V. Lokesha, Shwetha. B. S, Ranjini P. S , Cangul Ismail N and Cevik Ahmet S, "New bounds for Randic and GA indices", Journal of Inequalities and Applications, 180(1), 1-7, 2013.

[17] V. Lokesha, T. Deepika, P. S. Ranjini and I.N. Cangul, "Operation of nanostructures via $\mathrm{SDD}, \mathrm{ABC}_{4}$ and $\mathrm{GA}_{5}$ indices", Applied Mathematics and Nonlinear Sciences, 2(1), 173-180, 2017.

[18] Milicevic, S. Nikolic and N. Trinajstic, "On reformulated zagreb indices”, Mol. Divers., 8, 393-399, 2004.

[19] Randic. M, "On the characterization of molecular branching”, $J$. Am. Chem. Soc., 97, 6609-6615, 1975.

[20] Shwetha. B. Shetty, V. Lokesha, P.S. Ranjini, and K.C. Das, "Computing some topological indices of Smart polymer", Digest Journal of Nanomaterials and Biostructures, 7(3), 1097-1102, 2012.

[21] Shwetha. B. Shetty, V. Lokesha and P. S. Ranjini, "On The Harmonic Index of Graph Operations”, Transactions on Combinatorics, 4(4), 5-14, 2015.

[22] Shwetha. B. Shetty, V. Lokesha, P.S. Ranjini and Naci cangul, "Computing ABC, GA, Randic and Zagreb Indices", Enlightens of Pure and Applied Mathematics, 1(1), 17-18, 2015. 
[23] Shwetha. B. Shetty, V. Lokesha, A. Bayad and P. S. Ranjini, "A Comparative Study of Topological Indices and Molecular Weight of Some Carbohydrates", Journal of the Indian Academy of Mathematics, 34(2), 627-636, 2012.
[24] Vukicevic. D and M. Gaperov,” Bond additive modeling 1. Adriatic indices", Croatica chemica acta, 83(3), 243-260, 2010.

[25] Vukicevic. D, "Bond Additive Modeling 2. Mathematical properties of Max-min rodeg index”, Croatica chemica acta, 83(3), 261-273, 2010. 